\title{
Using Neural Network to Extract Knowledge from Database
}

\author{
Zhou yuanhui, Lu Yuchang, Shi Chunyi \\ Department of Computer Science, Tsinghua University \\ Beijing, 10084, P.R. of China
}

\begin{abstract}
Classification, which involves finding rules that partition a given data set into disjoint groups, is one class of data mining problems. Approaches proposed so far for mining classification rules for large databases are mainly decision tree based on symbolic learning methods. In this paper, we use artificial neural network to mine classification rules. We present a novel approach, called LBSB, composed of two phases to extract rules from artificial neural network and discover knowledge in databases. Some experiments have demonstrated that our method generates rules of better performance than the decision tree approach in noisy conditions.
\end{abstract}

\section{Introduction}

Although the stored data are a valuable asset of an organization, most organizations may face the problem of data rich but knowledge poor sooner or later. This situation aroused the recent surge of research interests in the area of data mining[1][2]. One of the data mining problems is classification. Classification is the process of finding the common properties among different entities and classifying them into classes. The results are often expressed in the form of the classification rules. The classification problem is to obtain a set of rules by using a given training data set. By applying these rules to the testing set, the rules can be checked whether they generalize well.

In this paper, we apply the neural network to mine classification rules from databases with focus on articulating the classification rules represented by the trained neural network. The reason is that compared with symbolic inductive learning such as C4.5, artificial neural networks perform better than in noisy condition. But the major weakness of this approach is that the knowledge learned by a neural network is very difficult to interpret. It is becoming increasingly apparent that without some form of explanation capability, the full potential of trained artificial neural networks may not be realized. How to explain the behavior of the trained neural networks, how to understand the concept representation formed by the network and how to make this black box problem transparency have attract many researchers to work on it.

We present a novel method including two phases to extract rules from the trained neural network. The first phase is to extract rules from hidden layer to output layer. In this phase, we use a learning method to identify the regions in the hidden activation space in order that all hidden vectors in them generate the activation of a given output larger than a certain threshold. The second phase is to extract rules between input layer and hidden layer in a searching method to select all rules so that all instances covered by them generate hidden activation vectors lying in these regions. Some experiments have been done to prove that the rules extracted in this method have many advantages on symbol learning method such as $\mathrm{C} 4.5[5]$. 


\section{Mining classification rules using $\mathbf{N N}$}

\subsection{Neural network learning}

A three layer neural network is trained in this step. The training phase aims to find the best set of weights for the network which allow the network to classify with a satisfactory level of accuracy. An initial set of weights are chosen randomly . Updating these weights is done by using information involving. The neural networks have fully-connected hidden units in a single layer. The number of hidden units used in each network is determined by cross-validation within the training set. For each training set, we reduce the number of the hidden units and use cross validation to pick the hidden units and use cross validation to pick the network that is to be trained on all of the data in the training set. This phase is terminated when the stopping criterion meet.

\subsection{Rule extracted from trained network in LBSB}

This process includes two algorithms: LB algorithm and SB algorithm. The LB algorithm is used to identify the regions of hidden activation space so that all hidden vectors within them belong to positive class. The SB algorithm is to search all feature combination to make the input instances covered by them lying in these regions.

\subsubsection{LB algorithm}

This algorithm is used to extract a set of valid regions, called hypercubes whose dimensions are determined by the number of hidden nodes, in order that all input instances which cause their corresponded hidden activation vectors within these hypercubes belong to same class. Here, a hidden activation vector is composed of all hidden nodes' outputs for an input example. We assume that the activation function is the sigmod function

$$
F(a)=1 /\left(1+e^{-\lambda a}\right)
$$

where $\lambda$ determines the steepness of the function and $\lambda>0$. The activation of unit $\mathrm{j}$ (a hidden or output unit) is calculated by

$$
O_{j}=F\left(\sum_{i} W_{j i} X_{i}-\theta_{j}\right)
$$

From (1), we can see that $F(a)$ is a monotonic function. The value of $F$ will increase as $a$ grows. From (2), we can see that the sensitivity pattern for input vector $X$ which is directly connected with the output node $O_{j}$ depends on the connected weight $W_{j i}$. If $W_{j i}$ is greater than zero, then $o_{j}$ will increase with $W_{j i}$ increasing and the sensitivity pattern of $X_{i}$ is ' + '. Otherwise, $o_{j}$ will decrease and sensitivity pattern of $X_{i}$ is "-". The sensitivity pattern do not change in the whole input space. This will give us a chance to easily extract a set of hypercubes which approximately represent the original valid space in which all examples belong to same class. The LB algorithm is briefly described below. 
examples, are trained positive examples.

Repeat

Step2: Using region clustering algorithm, according to the failure positive examples, continue to extract a set of valid hypercubes from the whole hidden activation space, in which all hidden activation vector generate a given output larger than a threshold.

Step3: Expand the size of these valid hypercubes, including upward expanding and downward expanding.

Step4: Randomly generate mpositive examples, which causes a given output larger than a threshold, calculate their corresponded hidden activation vectors and the select the failure positive examples which do not lie in the valid hypercubes from the m positive examples.

until stopping criterion meet.

Step5: Extract rules such as If $\left(H \in D_{1}\right)$ or...or $\left(H \in D_{l}\right)$ then positive class, where $H$ is the hidden vector, $D_{i}$ represents ith valid hypercube.

We use a region clustering algorithm to extract a set of valid hypercubes according to the sensitivity pattern of hidden vector. We check each hypercube by finding the hidden vector which generates the smallest activation of the given output. If the smallest activation of output is larger than a given threshold, all vectors in this hypercube will cause the output exceeding to the threshold. So this hypercube is a valid region. Else, there must be some hidden vectors in it that do not satisfy the condition and the hypercube is invalid. Because the sensitivity pattern of a hidden vector doesn't change, this point must be the topmost of the hypercube. According to the sensitivity pattern, we can easily to find this point. For example, a 3-dimentinal hypercube with three valid intervals $\left[a_{1}, b_{1}\right],\left[a_{2}, b_{2}\right],\left[a_{3}, b_{3}\right]$, the sensitivity pattern of the hidden vector is,,++- . Then the hidden vector which produces the smallest output is $\left(a_{1}, a_{2}, b_{3}\right)$. The region clustering algorithm is described as follow.

The region clustering algorithm

We start with $j_{\max }=1$

For all hidden activation vectors which are generated by the failure positive examples, do

begin

Step1: let $x$ be the hidden vector currently under test. If it is in a valid hypercube $D_{j}$, continue this loop.

Step2: Set $j=1$ and flag: $=$ NotYetFoud

Step3: While $j \leq j_{\max }$ and flag: $=$ NotYetFoun , do

begin

Step3-1: Select the smallest hypercube $D_{j}{ }^{\prime}$ so that it can contain the valid hypercube $D_{i}$ and $\{x\}$.

Step 3-2: Set Status: $=O K$

Step 3-3: According to the sensitivity pattern, we can select the vertex of hypercube $D_{j}{ }^{\prime}$, which causes the output smallest. If this output value is smaller than the given threshold, then set Status $=$ NotOK .

Step3-4: If Status $=O K$, then set flag= Foundand exit from this level of loop. 
Otherwise, increment $j$ as $j::=j+1$, and continue.

end.

Step4: If flag= Found,then $B_{:}:=D_{j}^{\prime}$. If flag= NotYetFound, then create a new set containing only $x$,i.e.

begin

Step4-1: $j_{\max }=j_{\max }+1$

Step4-2: Create $D_{j_{\max }}=\{x\}$

end.

End.

The extracted hybercubes maybe cover a part of the positive hidden activation space. So we can expand these hybercubes to cover the space as more as possible to describe the network to an satisfactory degree of accuracy. This can be done according to the sensitivity pattern. The expanding proceeding includes upward expanding and downward expanding. Upward expanding is to enlarge the size of a hypercube along the directions which cause the output activation increasing. Downward expanding begins from the topmost of the hypercube, which generates the smallest output, to enlarge the hypercube along the direction that causes the output activation decreasing and exceeding the given threshold in the mean time. The enlarging direction can be decided according to the sensitivity pattern of hidden vector. For the two expanding methods, there is a same condition that all expanding hypercubes can not intersect. This can avoid generating redundant rules.

The stopping criterion can be done in different ways, such as the number of iteration, the ratio of the number of the examples that lies in the hypercubes to that of the whole positive examples, etc. If the stopping criterion meets, then stop. Otherwise continue extracting the hypercubes and expanding them in our clustering learning according to the failure examples.

\subsubsection{The SB algorithm}

After LB algorithm has been used to extract a set of hypercubes, the SB algorithm heuristically searches through the rule space from input layer to hidden layer in terms of combinations of attributes so that all examples covered by the rules generate hidden activation vectors lying in the valid hypercubes. For a single hidden unit, there are several valid intervals extracted in the first phase. SB extracts rules for each hidden unit, which are expressed in terms of the units that feed into it so that all instances covered by them generate the activation of the hidden unit within the these valid intervals. SB is a searching algorithm which is similar to that of [6], but there are some differences. Different from other search-based algorithms, such as KT algorithm[4], this algorithm is based on a heuristic search which is conducted by combining the positive and negative weights. This algorithm first convert all negative weights to positive weights, then sort the weights in descend ordering and recursively extract all essential prime implicants(the maximum-general rules) by using the solution function and the bounding function in the depth-first backtracking tree search. The sorting of weights in descending order ensures that the search is done in an optimal order and non-prime implicants are not generated. The bounding function in the search process eliminates the possibility of generating any unnecessary subpath which cannot be a part of any solution subsets. The bounding function is given as 


$$
\sum_{j=1}^{k} w_{j} x_{j}+\sum_{j=k+1}^{n} w_{j} \geq T \quad \text { with } \sum_{j=1}^{k-1} w_{j} x_{j}<T .
$$

where $k$ is again the tree depth of a current subpath and $x_{j}$ is in $\{0,1\}$.

The use of the solution function in the tree search ensures that no unnecessary superset of any solution subset is generated. The solution function is given as follow:

$$
\sum_{j=1}^{k} w_{j} x_{j} \geq T \quad \text { with } \sum_{j=1}^{k-1} w_{j} x_{j}<T .
$$

where $k$ is the tree depth of a current subpath and $x_{j}$ is in $\{0,1\}$. The detail will see [6]. SB algorithm is different from backtrack tree algorithm on two points:

(a) SB may extract rules from a closed intervals, such as $a<\sum W_{i j} h_{i}<b$. When it generates all leaves, called positive leaves, which make $a<\sum W_{i j} h_{i}$, it will continue searching from these positive leaves and producing all leaves, called negative leaves that make $\sum W_{i j} h_{i}<b$. Then extract rules from the final negative leaves according to the binary code from root to these leaves..

(b) SB will extract rules from a set of intervals for each hidden node. In order to reduce the complexity of running time, we use only one backtrack tree to finish this searching. First, sort the intervals bounds in a increasing order and divide them into two groups, one for lower bound $a_{i} \in A$ and one for higher bound $b_{i} \in B$. Then search in this order to generate positive and negative leaves. When meeting the element of $\mathrm{A}$, then generating all positive leaves that cause the $a_{k}<\sum W_{i j} h_{j}$ in the method[6] and continue searching from these leaves. When meeting the element of $\mathrm{B}$, then generating all negative leaves that cause the $\sum W_{i j} h_{j}<b_{k}$ and extract rules according to the binary code from root to these negative leaves, and then continue searching from all positive leaves that cause the $b_{k}<\sum W_{i j} h_{j}$. This process continues until the largest bound has been searched.

\subsection{Rewriting the extracted rules}

After finishing the LB and SB algorithm, a rewriting module is used to rewrite rules containing some symbols designating hidden units which do not correspond to predefined attributes or concepts so that these symbols are eliminated from the rules. This process contains two phrases: one is to eliminate the symbols representing the hidden nodes and the other is to combine the rules extracted from all hidden nodes. This module make it easy for us to understand the extracted rules.

\section{Experiment and Results}

In this section, we describe the databases and representations used in the experiments. We compare the rules extracted in LBSB with those extracted from decision tree to see which performs better in noisy condition.

\subsection{The Databases and the Representations}

We use two databases, which are Splice junction and Pima Indians diabetes, from the 
University of California Irvine data repository for machine learning and one real-world database from YuXi cigarette factory.

¡aSplice junction: Splice junctions are points on a DNA sequence at which 'superfluous' DNA removed during the process of protein creation in higher organism. The problem posed in this database is to recognize, given a sequence of DNA, boundaries between exon and intron. The data set contains 3190 examples. We select 1500 examples for training and else for testing.

¡QPima Indians Diabetes: This database was made by National Institute of Diabetes and Digestive and Kidney Diseases. Several constraints were placed on the selection of these instances from a larger databases. In particular, all patients here are females at least 21 years old of Pima Indian heritage. There are total 768 instances, 500 for class 0 and 268 for class 1 . We use half of them for training.

joA real-world database: We use a real-world database--Information management and Decision support system of the YuXi cigarette factory. We use a large database on market information of this factory, analysis the relationship of the economic parameters and extract decision knowledge from the database to offer decision supporting for managers. In this problem, we use 32 economic attributes that may have influence on the sales volume of several brands of cigarette made by $\mathrm{YuXi}$ cigarette factory. We use 20 anmual data of these attributes and the classes are the sales volume of the high-grade, the middle grade and the low grade cigarette of the YuXi factory. By this, we hope to know which economic attributes have important influence on the sales volume and how they effects.

\subsection{Experiment and the result}

We compare the rules extracted from neural network in LBSB with those extracted from decision tree. The neural networks used for the three domains have fullyconnected hidden units in a single layer. The number of hidden units used in cach network is determined by cross-validation within the training set. For each training set, we reduce the number of the hidden units and use cross validation to pick the network that is to be trained on all of the data in the training set. Decision trees are also induced by these data and rules extracted from them.

In testing the tolerance to noise, we added noise at different levels by changing the class labels of training instances randomly. For example, a 10\% noise level means that we perturb $10 \%$ of the training instances by changing their class labels. We test it using the three databases. For example, in the Splice junction domain, we use 1500 trained examples for extracting. The rules extracted in LBSB achieved an error rate of $7.6 \%$ and $\mathrm{C} 4.5$ a rate of $9.3 \%$ in the absence of noise. At the $10 \%$ noisy level, the former gained an error rate of $9.1 \%$. whereas the latter yield $13.9 \%$. In the case of $20 \%$ noisy level, the former reached $11.7 \%$ error rate, whereas the latter got $19.4 \%$. 


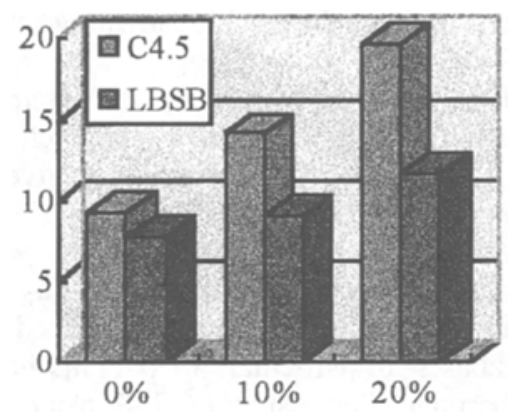

Fig. 1. The error rate comparison in the Splice Junction domain

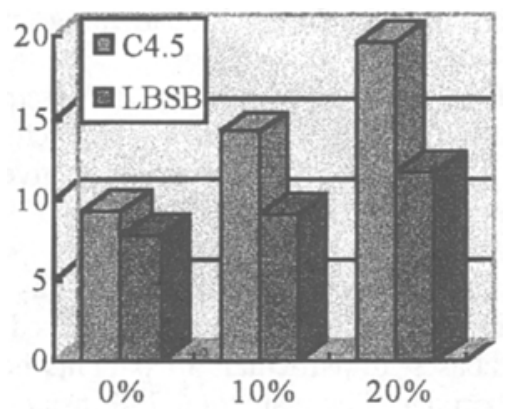

Fig. 2. The error rate comparison in the Sales Volume domain

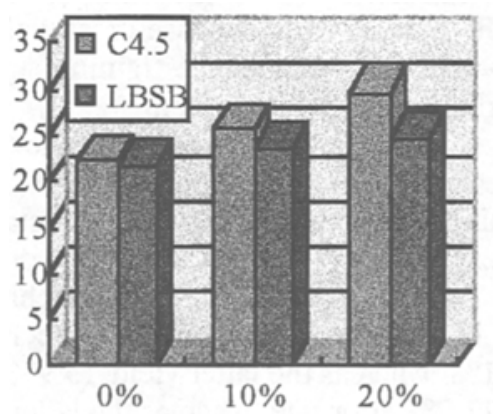

Fig .3. The error rate comparison in the Pima Indians Diabetes domain

In the Sales Volume domain, we use 360 positive examples for extracting. The rules extracted in LBSB achieved an error rate of $11.1 \%$ and $C 4.5$ a rate of $14.7 \%$ in the absence of noise. At the $10 \%$ noisy level, the former gained an error rate of $13.7 \%$,whereas the latter yield $17.2 \%$. In the case of $20 \%$ noisy level, the former reached $9.7 \%$ error rate, whereas the latter get $13.8 \%$.

From these experiments, we can see that in the noise condition, the error rate of the 
rules grows more quickly in C4.5[5] than in LBSB. This demonstrates that in the noisy condition the rules extracted from neural networks in LBSB have stronger tolerance ability than those extracted from decision trees. The main reason is that the neural network performs better in noisy condition than $\mathrm{C4.5}$. The results also show that using the neural network, high quality rules can be discovered from the databases.

\section{Discussion and conclusion}

In this paper, we apply the neural network to mine classification rules from databases with focus on articulating the rules represented by network. We present a novel approach to extract rules from the neural networks with distributed representation. Here we use the tolerance ability against noisy data to evaluate the quality of the rules extracted from the neural network.

The resistant against noisy data is very important because in the real-would problem, there may be some noisy data. From the experiment, we can see that compared with the rules extracted from decision trees, the rules extracted from neural networks in LBSB can perform better in noisy condition. This demonstrates the advantage of neural network over decision tree in data mining.

There are also some future works to do: (a)how to efficiently represent the extracted rules. LBSB extracts only if-then rules. But for some problems, it will take a long time and generates many such kinds of rules to cover positive hidden activation space. The future work is to extract rule described by a more powerful representation to reduce the number of the rules.(b) how to reduce the number of rules' conditions.

\section{Bibliography}

[1] Ming-Syan Chen, Jiawei Han and Philip S.Yu: Data Mining: An Overview from Database Perspective. Technology Report, 1996.

[2] Victor Ciensielski and Gregory Palstra: Using a Hybrid Neural / Expert System for Data Base Mining in Market Survey Data. In Proc. 1996 International Conference on Data Mining and Knowledge Discovery(KDD 96), Portland, Oregon, August, 1996.

[3] Mark W.Craven and Jude W.Shavlik: Using Sampling and Queries to Extract Rules from Trained Neural Networks. Proceeding of the Eleventh International conference in Machine Learning, Morgan Kaufmann, San Francisco, CA, 1994.

[4] LiMin Fu: Rule Generation from Neural Networks. IEEE Transactions On Systems, Man and Cybernetics, Vol.24,NO.8, August 1994.

[5] J.R.Quinlan: C4.5: Programs for Machine Learning. Morgan Kaufmann,1993

[6] Ishwar K.Sethi and Jae H.Yoo: Symbolic approximation of feedforward neural network. In Pattern Recognition in Practice IV, pp 313-324, North-Holland, 1994. 